Pontifí́ia Universidade Católica $_{\text {a }}$ DO RIO DE JANEIRO

Marco Antonio Pérez Rosas

\title{
Avaliação de Modelos do Comportamento de Reparos do Tipo Luva em Dutos com \\ Defeitos de Perda de Espessura
}

Tese de Doutorado

Tese apresentada ao Programa de Pósgraduação em Engenharia Mecânica da PUC-Rio como requisito parcial para obtenção do título de Doutor em Engenharia Mecânica.

Orientador: Prof. José Luiz de França Freire

Rio de Janeiro

Agosto de 2010 
Pontifícia U UIVERSIDAde Católica $_{\text {a }}$ DO RIO DE JANEIRO

Marco Antonio Pérez Rosas

\begin{abstract}
Avaliação de Modelos do Comportamento de Reparos do Tipo Luva em Dutos com

Defeitos de Perda de Espessura
\end{abstract}

Tese apresentada como requisito parcial para obtenção do grau de Doutor pelo Programa de Pósgraduação em Engenharia Mecânica do Centro Técnico Científico da PUC-Rio. Aprovada pela Comissão Examinadora abaixo assinada.

Prof. José Luiz de França Freire

Orientador

Departamento de Engenharia Mecânica - PUC-Rio

Prof. Carlos Alberto de Almeida

Departamento de Engenharia Mecânica - PUC-Rio

Prof. Luiz Cláudio de Marco Meniconi CENPES/PETROBRAS

Profa. Ivani de Souza Bott

Departamento de Engenharia de Materiais - PUC-Rio

Prof. Antonio Lopes Gama Universidade Federal Fluminense

Prof. José Eugenio Leal Coordenador Setorial do Centro Técnico Científico - PUC-Rio 
Todos os direitos reservados. É proibida a reprodução total ou parcial do trabalho sem autorização da universidade, do autor e do orientador.

\section{Marco António Pérez Rosas}

Graduou-se em Engenharia Mecânica Universidad Nacional San Agustín de Arequipa - Perú em 2004. Mestre em Engenharia Mecânica pela Pontifícia Universidade Católica do Rio de Janeiro em 2006.

Ficha Catalográfica

Rosas, Marco Antonio Pérez

Avaliação de modelos do comportamento de reparos do tipo luva em dutos com defeitos de perda de espessura / Marco Antonio Pérez Rosas; orientador: José Luiz de França Freire.- 2010

v., 196 f.: il. (color) ; $30 \mathrm{~cm}$

Tese (doutorado) - Pontifícia Universidade Católica de Rio de Janeiro, Departamento de Engenharia Mecânica, 2010.

Inclui bibliográficas.

1. Engenharia mecânica - Teses. 2. Reparo. 3. Duto. 4. Camadas metálicas. 5. Material compósito. 6. Defeitos. 7. Perda de espessura. 8. Teste hidrostático. 9. Elementos finitos. I. Freire, José Luiz de França. II Pontifícia Universidade Católica do Rio de Janeiro. Departamento de Engenharia Mecânica. III. Título. 
Al Señor Jesucristo, mi mamá Nancy, mi querida familia y mis valiosos amigos 


\section{Agradecimentos}

Ao professor José Luiz de França Freire e ao professor Ronaldo Domingues Vieira, pela paciência e orientação durante o desenvolvimento do curso de mestrado.

A meus pais, Nancy e Saúl, irmãos, Saúl, Guillermo e Carlos, avô don Guillermo e doña Isolina, e de mais familiares.

A Fernanda, pelo seu amor, carinho e paciência.

A meus amigos e sócios Léo e Jesús.

A meus amigos e colegas de laboratório, Maira, Luana, Sabrina, Gilmar, Jorge, Guillermo, Cristian, Hugo, Jaime que sem sua ajuda não teria concluído este trabalho, em especial ao sempre solicito Gerardo.

A meus amigos Jean, Adenilson e Clevson, companheiros de apartamento.

Aos professores da PUC-Rio pelo ensino.

Ao grande Sr. Garcia (viga) pela sua lição de vida.

A todos os colegas da pós-graduação.

Ao Departamento de Engenharia Mecânica da PUC-Rio e seus funcionários, pela colaboração para comigo.

A todas aquelas pessoas que de alguma outra forma participaram no desenvolvimento da tese. 


\section{Resumo}

Pérez Rosas, Marco Antonio; Freire, José Luiz de França. Avaliação de Modelos do Comportamento de Reparos Tipo Luva em Dutos com Defeitos de Perda de Espessura. Rio de Janeiro, 2010. 196p. Tese de Doutorado - Departamento de Engenharia Mecânica, Pontifícia Universidade Católica do Rio de Janeiro.

Devido ao seu alto custo e à sua importância estratégica, a busca por soluções que estendam a vida útil das redes dutoviárias é plenamente justificável. Uma vez detectado um defeito, procura-se restabelecer sua capacidade operacional, reparando a seção danificada. A maioria dos estudos está orientada a determinar o comportamento de um duto reparado utilizando técnicas numéricas e/ou experimentais. Outros apresentam equações simples para o cálculo da espessura de reparo tentando garantir a segurança na operação, porém sem demonstrar o comportamento do mesmo. Neste trabalho foi desenvolvida uma metodologia analítica para determinação do comportamento de dutos reparados por luvas metálicas coladas ou de material compósito. Esta metodologia permite também conhecer as pressões que ocasionarão o escoamento e falha, seja no duto ou no reparo. Acredita-se que com a utilização desta metodologia o projeto de um reparo será mais eficiente, permitindo escolher melhor o material e a espessura de reparo, segundo as exigências de cada projeto. São apresentados resultados de simulações numéricas com o propósito de conhecer o comportamento, otimizar o dimensionamento e avaliar os resultados obtidos analiticamente para estes tipos de reparos. Por fim, é apresentado um estudo de caso, resultados de testes experimentais e um exemplo de aplicação para a determinação da espessura de reparo. Com isto foi possível conhecer os comportamentos não estudados numericamente, e mostrou-se que, o controle das deformações no defeito dependerá do módulo de elasticidade e da espessura do reparo, como era esperado.

\section{Palavras - chave}

Reparo; duto; camadas metálicas; material compósito; defeitos; perda de espessura; teste hidrostático; elementos finitos 


\section{Abstract}

Pérez Rosas, Marco Antonio; Freire, José Luis de França (Advisor). Evaluation of Sleeve Type Repair Models for Pipelines with Thickness Loss Defects. Rio De Janeiro, 2010. 196p. Doctoral Thesis Departamento de Engenharia Mecânica, Pontificia Universidade Catolica de Rio de Janeiro.

Due to their high cost and strategic importance, the search for solutions to extend the life cycle of pipeline networks is fully justifiable. Once a defect is detected, an attempt is made to restore operational capacity, repairing the defective section. Most studies are aimed at determining the behavior of a repaired pipe by using numerical and/or experimental techniques. Others present simple equations for the calculation of repair thickness trying to ensure a safe operation, yet without demonstrating the behavior of the pipe. In this study, an analytical methodology has been developed to determine the behavior of pipes repaired by attached metal sleeves or sleeves of composite material. This methodology also enables to know the stresses which may cause leakage and failure, be it on the pipe or on the repair area. It is believed that by using this methodology the project of a repair would be more efficient, enabling a better choice of repair material and thickness, according to the requirements of each project. Numerical simulation results are presented with the purpose of knowing the behavior, optimizing the sizing and assessing the results obtained analytically for this kind of repairs. Finally, a case study is presented, with the results of experimental tests and an example of application for determining repair thickness. This way it was possible to know behaviors that had not been studied numerically, and it was demonstrated that the control of defect deformations will depend on the elastic modulus and thickness of the repair, as was expected.

\section{Keywords}

Repair; pipeline; pipe; multilayers; epoxy resin; loss of thickness; corrosion; adhesive; hydrostatic test; extensiometria; finite elements. 


\section{Sumário}

1. Introdução 21

1.1. Objetivo 21

1.2. Considerações Iniciais $\quad 21$

1.3. Trabalhos Anteriores 22

1.4. Motivação 27

1.5. Roteiro da Dissertação 28

2. Fundamentação Teórica 29

2.1. Reparos para Dutos 29

2.1.1. Tipos de Reparos $\quad 30$

2.2. Normas para Projeto de Reparos de Material Compósito 45

2.2.1. Norma ISO/TS $24817 \quad 46$

2.2.2. Norma ASTM PCC-2 49

2.2.3. Outras Referências 50

2.3. Aspectos Básicos do Emprego de Adesivos 51

2.3.1. Junta Colada 51

2.3.2. Projeto de Junta 52

2.3.4. Tratamento Superficial 53

2.3.5. Adesivos Estruturais $\quad 55$

2.3.6. Seleção de Adesivos 56

2.4 Resistência de Materiais $\quad 57$

2.4.1. Elasticidade em Cilindros 57

2.4.2. Noções Básicas da Plasticidade 59

2.4.3. Tensões em Dutos sem Defeitos 62

2.4.4. Equações Semi-Empíricas para Duto com Defeitos de 63 Corrosão

2.5. Avaliação de Componentes Estruturais Utilizando o Critério 65 RSF da API 579 
3. Modelagem Numérica 67

3.1. Introdução 67

3.2. Detalhes dos Modelos Desenvolvidos 68

3.3. Variação da Espessura de reparo 74

3.3.1. Comparação de resultados 75

3.4. Variação do Comprimento do Defeito 80

3.5. Variação do Comprimento do Reparo 83

3.5.1. Reparo de Defeitos Longos com Reparos Adjacentes 89

3.6. Simulação de um Duto Reparado com Material Compósito 90

4. Modelagem Analítica 92

4.1. Descrição do Problema 92

4.2. Modelagem Analítica do Reparo de um Duto com Defeito 92

4.2.1 Determinação das equações que governam o 92 comportamento de um duto reparado

4.2.2 Aplicação das equações nas regiões de comportamento 98 elasto-plástico de um duto reparado

4.2.3 Comparação dos Resultados 111

4.3. Reparo para Defeitos com Comprimento não Infinito 113

4.4. Formulação para Reparos Aplicados em Dutos Ativos 124

4.5. Formulação para Reparo de Material Compósito 131

4.5.1 Comparação dos Resultados 140

4.5.2 Recomendações para Projeto de Reparos de Material 141 Compósito

5. Estudos de Caso e Aplicação 142

5.1. Estudo de Caso : Testes Experimentais em Dutos em 142

Escala Real

5.1.1 Comparação Numérica - Experimental 149

5.1.2 Comparação Numérica - Analítica 151

5.2. Testes Experimentais em Dutos em Escala Reduzida 152 
6. Conclusões

Bibliografia

Anexos 179

$\begin{array}{ll}\text { A. Adesivos } & 179\end{array}$

B. Formulações do Modelo Analítico 179

C. Dispositivo Experimental 194

D. Comparações Adicionais 196 


\section{Lista de Figuras}

Figura 2.1 - Processos na instalação de um novo trecho de duto

Figura 2.2 - Defeito interno reparado com deposição de solda externa

Figura 2.3 - Preenchimento da perda do material na superfície do duto a ser reparado

Figura 2.4 - Reparo de dupla calha soldada tipo A

Figura 2.5 - Reparo de mangas enchidas de epóxi

Figura 2.6 - Reparo de dupla manga soldada tipo B

Figura 2.7 - Variantes do reparo de dupla calha soldada tipo

B

Figura 2.8 - Comparação entre as resistências mecânicas de fibras comerciais

Figura 2.9 - Tipos de material compósito formado por fibra de vidro

Figura 2.10 - Curva de resistência vs tempo nos materiais compósitos

Figura 2.11 - Reparos utilizando a marca comercial Armor Plate Pipe Wrap

Figura 2.12 - Abraçadeiras mecânicas

Figura 2.13 - Hot tapping

Figura 2.14 - Tipos de falhas nas juntas coladas

Figura 2.15 - Tipos de carregamentos

Figura 2.16 - (i) Equilíbrio de forças num ponto. (ii)

Deslocamentos e deformações num ponto

Figura 2.17 - Estudo incremental

Figura 3.1 - Elementos 3D

Figura 3.2 - Curvas bilineares tensão vs deformação 
Figura 3.3 - Comparação entre modelos de material BE e ME

Figura 3.4 - Curvas de material BE e ME 71

Figura 3.5 - Dimensões dos modelos numéricos 72

Figura 3.6 - Dimensões dos modelos numéricos 73

Figura 3.7 - Condições de contorno 73

Figura 3.8 - Relação quando os nós da espessura da parede alcança o Su

Figura 3.9 - Modelo analisado no ANSYS v.11.0 74

Figura 3.10 - Comprimento e distribuição das camadas metálicas

Figura 3.11 - Comparação entre dutos reparados com defeitos de 40 e $120 \mathrm{~mm}$ de comprimento, utilizando reparo de $2 \mathrm{~mm}$ de espessura em ambos os casos:

Figura 3.12 - Comparação entre modelos com defeitos de 40 e $80 \mathrm{~mm}$ de comprimento, utilizando o reparo de $1 \mathrm{~mm}$ de espessura em ambos os casos

Figura 3.13 - Comprimento e distribuição das camadas metálicas. À direita mostra-se o reparo com extremos escalonados

Figura 3.14 - Distribuição das tensões normalizadas vM para uma pressão de $10 \mathrm{MPa}$ (defeito de $40 \mathrm{~mm}$ )

Figura 3.15 - Distribuição das tensões normalizadas vM a uma pressão de $10 \mathrm{MPa}$ (defeito de $40 \mathrm{~mm}$ ).

Figura 3.16 - Modelo de duto com defeito e sem reparo

Figura 3.17 - Distribuição das tensões circunferenciais normalizadas

Figura 3.18 - Tensões cisalhantes máximas no adesivo

Figura 3.19 - Modelos com e sem reforço entre as mangas adjacentes

Figura 3.20 - Comprimento e distribuição das camadas metálicas

Figura 3.21 - Tensão von Mises variando com a pressão interna no duto reparado com material compósito 
Figura 4.1 - Distribuição das tensões na parede do duto reparado (*calculada utilizando os elementos finitos)

Figura 4.2 - Modelos de materiais. Reparos fabricados com material compósito serão considerados na seção 4.5

Figura 4.3 - Regiões do comportamento

Figura 4.4 - Possíveis comportamentos de um duto com reparo metálico

Figura 4.5 - Estado de tensões e deformações num ponto do defeito de um duto 102

Figura 4.6 - Erro nas aproximações das tensões von Mises 104 Figura 4.7 - Aplicação geral de incrementos de carga 105 Figura 4.8 - Aplicação geral de incrementos de carga 2 Figura 4.9 - Aplicação de incrementos num duto reparado 107 Figura 4.10 - Efeito da variação da espessura do reparo e pressões que originam o escoamento e a falha dos espécimes calculadas analiticamente

Figura 4.11 - Comparação dos comportamentos dos modelos numéricos e analíticos

Figura 4.12 - Modelo com defeito de $40 \mathrm{~mm}$ de comprimento

Figura 4.13 - Modelo numérico de um duto com defeito e sem reparo

Figura 4.14 - Comparação dos resultados numérico-analíticos de um duto com defeito e sem reparo

Figura 4.15 - Esquema do comportamento de um duto com defeito e sem reparo

Figura 4.16 - Comparação entre as simulações numéricas e as equações desenvolvidas para um duto com defeito e sem 123 reparo

Figura 4.17 - Efeito da variação do comprimento do defeito para um duto reparado com $1.5 \mathrm{~mm}$ de espessura de reparo 124

Figura 4.18 - Comportamento de um duto reparado a uma pressão interna $\mathrm{Pi}$ 
Figura 4.19 - Aplicação de incrementos num duto reparado com pressão interna

Figura 4.20 - Possíveis comportamentos de um duto com reparo metálico e com pressão interna na instalação diferente de zero

Figura 4.21 - Comparação entre um duto reparado a uma pressão interna de $4 \mathrm{MPa}$ e outro reparado sem pressão interna. O sinal " $\backslash$ " significa paralelismo para as retas

Figura 4.22 - Efeito da variação na pressão de instalação do reparo

Figura 4.23 - Interferência de cilindros

Figura 4.24 - Modelos de materiais para duto reparado com material compósito

Figura 4.25 - Regiões do comportamento de um duto reparado com material compósito

Figura 4.26 - Possíveis comportamentos de um duto com reparo de material compósito e com pressão interna na instalação que pode ser diferente de zero

Figura 4.27 - Comparação dos resultados das simulações numéricas com o modelo analítico proposto

Figura 5.1 - Dimensões dos espécimes tubulares 143

Figura 5.2 - Localização dos extensômetros

Figura 5.3 - Deformações na região do defeito para o reparo de fibra de carbono

Figura 5.4 - Deformações corrigidas na região do defeito para o reparo da fibra de carbono

Figura 5.5 - Comparação das deformações no defeito para os espécimes reparados com fibra de carbono e fibra de vidro

Figura 5.6 - Comparação das deformações na janela e no defeito fora da janela nas diferentes camadas de reparo

Figura 5.7 - Comparação das deformações na parede do duto

Figura 5.8 - Comparação das deformações na $9^{\circ}$ camada de reparo 
Figura 5.9 - Deformações nas diferentes camadas do reparo localizadas na janela do defeito

Figura 5.10 - Junção da figura 5 na figura 1

Figura 5.11 - Curva discretizada do material do duto utilizado

Figura 5.12 - Comparação Numérico -experimental 150

Figura 5.13 - Comparação Numérica - analítica

Figura 5.14 - Aplicação do reparo de chapa metálica

Figura 5.15 - Dimensões dos espécimes tubulares reparados com chapa metálica: método $A$ e $B$

Figura 5.16 - Gráfico volume de água injetado vs pressão interna

Figura 5.17 - Instrumentação do espécime tubular

Figura 5.18 - Deformações vs pressão interna no espécime reparado com material compósito

Figura 5.19 - Comparação das deformações no reparo de chapa metálica e material compósito

Figura 5.20 - Gráfico pressões vs deformações adimensionais

Figura 5.21 - Comportamento do duto reparado com o material compósito, fibra de vidro e de carbono

Figura 5.22 - Comportamento do duto reparado com chapas metálicas coladas: chapa de baixo carbono, de material igual ao duto e de alta resistência

Figura C.1 - Esquema do sistema de pressão

Figura C.2 - Sistema de aquisição de dados

Figura D.1 - Comparação das deformações no defeito e fora do reparo

Figura D.2 - Comparação Numérico -experimental considerando a $3^{\circ}$ e $9^{\circ}$ camada do reparo localizadas a $90^{\circ}$ da janela do defeito 


\section{Lista de Tabelas}

Tabela 2.1 - Classes para reparos de material compósito

Tabela 2.2 - Documentação requerida por norma para cada classe de reparo

Tabela 2.3 - Propriedades de testes requeridos segundo norma

Tabela 2.4 - Fatores importantes ao selecionar-se um adesivo

Tabela 2.5 - Métodos para cálculo da pressão de operação nos dutos com defeito

Tabela 3.1 - Propriedades mecânicas do duto e chapa bilinear

Tabela 3.2 - Propriedades mecânicas do duto e chapa multilinear

Tabela 3.3 - Pressões que originam o escoamento e a falha dos modelos

Tabela 3.4 - Comparação entre as pressões de escoamento e de ruptura obtidas numérica e experimentalmente

Tabela 3.5 - Pressões que originam o escoamento e a falha dos modelos

Tabela 3.6 - Pressões que originam o escoamento e a falha nos modelos numéricos

Tabela 3.7 - Tensões e deformações no centro dos defeitos a $\mathrm{P}=18 \mathrm{MPa}$

Tabela 3.8 - Tensões e deformações no centro dos defeitos a $\mathrm{P}=18 \mathrm{MPa}$ (2)

Tabela 3.9 - Propriedades do material compósito

Tabela 4.1 - Propriedades mecânicas utilizadas no modelo analítico

Tabela 4.2 - Propriedades geométricas utilizadas no modelo 
analítico

Tabela 4.3 - Comparação entre as pressões de escoamento e de ruptura obtidas analiticamente, numérica e experimentalmente

Tabela 4.4 - Métodos para o cálculo de M

Tabela 4.5 - Comparações entre modelos analíticos e numéricos $(\mathrm{L}=40 \mathrm{~mm}, \mathrm{e}=1 \mathrm{~mm})$

Tabela 4.6 - Efeito do parâmetro 1-d/tM nos resultados analíticos $(\mathrm{L}=40 \mathrm{~mm}, \mathrm{e}=1 \mathrm{~mm})$

Tabela 4.7 - Comparações entre modelos analíticos e numéricos $(\mathrm{L}=120 \mathrm{~mm}, \mathrm{e}=1.5 \mathrm{~mm})$

Tabela 4.8 - Efeito do parâmetro 1-d/tM nos resultados analíticos $(\mathrm{L}=120 \mathrm{~mm}, \mathrm{e}=1.5)$

Tabela 4.9 - Comparações entre modelos analíticos e numéricos $(\mathrm{L}=80 \mathrm{~mm}, \mathrm{e}=1.5 \mathrm{~mm})$

Tabela 4.10 - Efeito do parâmetro 1-d/tM nos resultados analíticos $(\mathrm{L}=80 \mathrm{~mm}, \mathrm{e}=1.5)$

Tabela 5.1 - Propriedades do material compósito

Tabela 5.2 - Propriedades do material do duto utilizado

Tabela 5.3 - Propriedades geométricas e dos materiais

Tabela 5.4 - Resultados dos testes nos dutos reparados com chapa metálica

Tabela 5.5 - Propriedades das resinas epóxi utilizadas segundo fornecedores

Tabela 5.6 - Propriedades da fibra de carbono segundo fornecedor

Tabela 5.7 - Características dos espécimes tubulares

Tabela 5.8 - Resultados dos testes nos dutos reparados com material compósito.

Tabela 5.9 - Características do espécime tubular

Tabela 5.10 - Características dos espécimes tubulares

Tabela 5.11 - Comparação dos resultados: reparo de chapa metálica e reparo de material compósito 
Tabela 5.12 - Propriedades da fibra de vidro e carbono

164

Tabela 5.13 - Propriedades das chapas metálicas 165

Tabela A.1 - Adesivos epóxis encontrados na indústria 179

Tabela A.2 - Adesivos epóxis híbridos $\quad 180$

Tabela A.3 - Adesivos anaeróbicos, acrílicos modificados e poliuretanos.

180

Tabela A.4 - Propriedades de adesivos estruturais utilizados na colagem de metais 


\section{Lista de Símbolos}

$P_{e q}$ : pressão de projeto (no caso de não existir outros carregamentos adicionais)

$P_{s}$ : pressão de defeito (máxima pressão de defeito admissível).

$\sigma_{e q}^{e}:$ tensão equivalente no regime elástico

$\sigma_{i}:$ tensão na direção principal "i”"

$e_{i}:$ deformação na direção principal “i”

$\Delta e_{i}^{p}$ :incremento de deformação plástica na direção "i”"

$S_{y}:$ limite de escoamento do material

$S_{u}:$ limite de ruptura do material

$S_{\text {flow }}$ :resistência ao colapso plástico

$A$ : área longitudinal de material perdido

$A_{0}$ : área longitudinal da seção com defeito

$M$ : fator de Folias

$L_{D C}$ : carga limite ao colapso plástico do componente danificado.

$L_{U C}$ : carga limite ao colapso plástico do componente sem defeito.

MAWP: máxima pressão de operação admissível, determinada pelo código do projeto.

$M A W P_{r}$ : máxima pressão de operação admissível do componente com defeito.

$\rho_{c}:$ densidade do compósito

$\rho_{m}:$ densidade da resina o matriz

$\rho_{f}:$ densidade da fibra

$f_{f}$ : fração volumétrica de fibra

$f_{m}$ : fração volumétrica de resina o matriz

$E_{d}:$ módulo de elasticidade do duto

$E_{r}:$ módulo de elasticidade do reparo

$E_{c}$ : módulo de elasticidade do adesivo

$E_{m}$ :módulo de elasticidade da mescla (reparo-adesivo)

$E_{m t}$ : módulo de elasticidade tangencial da mescla reparo-adesivo

$E_{m r}$ : módulo de elasticidade radial da mescla reparo-adesivo

$E_{t}$ : módulo de elasticidade tangencial do reparo

$\mu_{d}$ : coeficiente de Poisson do duto 
$\mu_{r}:$ coeficiente de Poisson do reparo

$\mu_{c}$ : coeficiente de Poisson do adesivo

$\mu_{m t r}$ : coeficiente de Poisson tangencial radial da mescla reparo-adesivo

$\sigma_{\text {circ d }}$ : tensão circunferencial no duto

$\sigma_{\text {circ } r}$ : tensão circunferencial no reparo

$\sigma_{\text {circ } c}:$ tensão circunferencial no adesivo

$P_{1}:$ pressão interna inicial

$P_{o}$ : pressão de interferência entre o duto e o adesivo

$P_{a}:$ pressão de interferência entre o adesivo e o reparo

$t:$ espessura da parede do duto

t.C : profundidade do defeito

$h:$ profundidade do defeito

$r$ : raio interno do duto

$L$ : comprimento do defeito

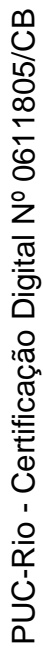

$\sigma_{c}:$ tensão circunferencial

$\sigma_{v M}$ : tensão de von Mises 